\title{
DETERMINANTES DO DESEMPREGO E INATIVIDADE DE JOVENS NO BRASIL METROPOLITANO ${ }^{1}$
}

\author{
Dênis Antônio da Cunha \\ Aracy Alves de Araújo \\ João Eustáquio de Lima ${ }^{4}$
}

\begin{abstract}
Resumo: Desemprego e inatividade são fenômenos que afetam distintamente a força de trabalho conforme as várias características pessoais, familiares e demográficas. Em comparação com as demais faixas etárias, os jovens moradores de regiões urbanas têm sido os mais atingidos. Nesse contexto, este estudo pretende verificar que fatores são mais relevantes para explicar o desemprego e a inatividade de jovens entre 16 e 29 anos de idade no Brasil metropolitano. Foi estimado um modelo logit multinomial a partir de dados da Pesquisa Nacional por Amostra de Domicílios - PNAD 2007, considerando as três categorias em que o jovem poderia se encontrar: inativo, ativo e empregado e ativo e desempregado. Os resultados indicaram que a probabilidade de inatividade é maior nos jovens que estudam e ocupam a posição de cônjuge na família; já a probabilidade de desemprego tende a diminuir à medida que aumenta a experiência dos indivíduos.
\end{abstract}

Palavras-chave: desemprego, inatividade, jovens, regiões metropolitanas, logit multinomial.

Recebido em:

13/9/11; Aceito em: 22/11/2011.

2 Doutor em Economia Aplicada.

Professor Adjunto do Departamento de Economia Rural da Universidade Federal de Viçosa.

E-mail: denisufv@gmail.com

3 Doutora em Economia Aplicada. Professora Adjunta do Departamento de Ciências Econômicas e Quantitativas da Universidade Federal do Piauí. Email: aracy.araujo@gmail.com

4 PhD. Em Economia Rural. Professor Titular do Departamento de Economia Rural da Universidade Federal de Viçosa.E-mail: jelima@ufv.br 
Abstract: Unemployment and inactivity are phenomena that affect the workforce in a distinct way, conform to several personal characteristics, families and demographics. Compared with other age groups, young residents of urban areas have been the most reached. Therefore, this study intends to investigate which factors are most relevant to explain unemployment and inactivity of young people between 16 and 29 years old in the metropolitan Brazil. It has been estimated a multinomial logit model based on data from the Pesquisa Nacional por Amostra de Domicílios - PNAD 2007, considering the three categories in which the young people could be meet: inactive, active and employed, active and unemployed. The results indicated that the probability of inactivity is higher among young people that study and held the position of spouses in the family; however the probability of unemployment tends to decrease when the individual experience increases.

Keywords: Unemployment, inactivity, young people, metropolitan areas, multinomial logit.

\section{Introdução}

No Brasil, a taxa de desemprego vem caindo nos últimos anos como revelam os dados da Pesquisa Nacional por Amostra de Domicílios PNAD. Entre 2001 e 2009, houve aumento no nível de ocupação em praticamente todos os setores da economia, sendo que a taxa média de desemprego no Brasil em 2010 foi de 6,7\%, a menor em oito anos (IPEA, 2008, 2010). Entretanto, uma taxa de desemprego decrescente ou baixa no agregado pode ser consistente com desemprego elevado para grupos específicos da população. Isso ocorre porque o desemprego é um fenômeno complexo, que afeta distintamente a força de trabalho, conforme as várias características pessoais, familiares e demográficas, assim como varia entre os diversos setores da economia (FERNANDES; PICCHETTI, 1999; ZYLBERSTAJN; BALBINOTTO NETTO, 1999).

A realidade do desemprego no Brasil mostra que este é um fenômeno que atinge, principalmente, jovens (de 15 a 29 anos) $)^{5}$, moradores de regiões urbanas, em comparação com as demais faixas etárias. Esta também é

Segundo o IPEA (2008), no âmbito das políticas públicas, a adoção desse recorte etário é bastante recente, já que antes era considerada jovem a população na faixa etária de 15 a 24 anos. A ampliação para 29 anos não é uma particularidade do Brasil, mas uma tendência geral de países que buscam instituir políticas para a juventude. Há duas justificativas principais para essa mudança: maior expectativa de vida para a população, em geral, e maior difículdade dessa geração em ganhar autonomia, em função das alterações no mercado de trabalho. 
uma realidade de outros países, inclusive dos Estados Unidos e diversas nações europeias, como apontam os estudos de Layard et al. (1991) e Korenman e Neumark (1997).

Segundo dados do IPEA (2008), em 2007, 4,6 milhões de jovens estavam desempregados, representando cerca de $63 \%$ dos desempregados no país. A taxa de desemprego juvenil foi cerca de três vezes superior à dos adultos: de $14 \%$ para os jovens e de 4,8\% para os adultos. Os dados apresentados por Pochmann (2007) também indicam que o desemprego tem aumentado mais entre os jovens em relação a outras faixas etárias. Segundo este autor, a taxa de desemprego de indivíduos entre 15 e 24 anos variou oito pontos percentuais, de $11,4 \%$ para $19,4 \%$, de 1995 para 2005, respectivamente, enquanto para o restante da população economicamente ativa (PEA), a variação foi de 1,9 pontos percentuais de $4,3 \%$ para 6,2\%. Ademais, conforme o IPEA (2010), entre 2001 e 2009 , enquanto indivíduos com mais de 24 anos passaram a participar mais da força de trabalho, houve diminuição da participação dos mais jovens, tendo sido a queda mais intensa para o grupo de 15 a 24 anos de idade, que registrou um decréscimo de 3,6 pontos percentuais no período.

Os jovens em idade de trabalhar constituem um dos segmentos mais vulneráveis na busca por um posto de trabalho, uma vez que, em geral, concorrem com pessoas de maior experiência profissional. Além disso, as altas taxas de inatividade, principalmente entre os de 16 a 17 anos, podem ser explicadas pelo maior tempo dedicado à educação, resultante do maior acesso ao ensino público e devido à preocupação em aumentar a formação profissional (CACCIAMALI; BRAGA, 2003; DIEESE, 2006).

O problema torna-se mais complexo para indivíduos com atributos pessoais específicos. $\mathrm{O}$ acesso dos jovens a melhores oportunidades de ingresso no mercado de trabalho, muitas vezes, é diferenciado com relação a gênero, cor e/ou raça, condição econômica da família e região de domicílio (BRAGA; RODARTE, 2005).

Nesse contexto, este estudo pretende verificar que fatores são mais relevantes para explicar o desemprego e a inatividade de jovens, entre 
16 e 29 anos de idade, nas principais regiões metropolitanas do Brasil. Serão testadas duas hipóteses:

i. A razão fundamental do desemprego é incerteza a respeito da qualidade do trabalhador, dado que, em geral, jovens têm baixa experiência e suas características produtivas não estão bem definidas para os empregadores ${ }^{6}$; $\mathrm{e}$

ii. A principal explicação para a inatividade é que os jovens estão fora da PEA, pois se dedicam sobretudo ao estudo.

A partir da resposta a essas questões de pesquisa, este estudo pretende contribuir para o aumento do conhecimento a respeito do mercado de trabalho brasileiro. Por um lado, se a primeira hipótese for confirmada, ela pode fornecer subsídios, conforme argumentam Camargo e Reis (2005), para que as instituições que regulam o mercado de trabalho tornem o processo de difusão de informações entre trabalhadores e empregadores mais simétrico. Por outro lado, a confirmação da segunda hipótese poderia estar indicando a superação da ideia de que, em países em desenvolvimento, o ingresso precoce no mercado de trabalho está ligado à menor frequência dos jovens à escola.

Ademais, de modo mais geral, conforme Zylberstajn e Balbinotto Netto (1999), é importante conhecer as razões pelas quais os indivíduos se tornam desempregados com vistas a indicar as medidas de políticas públicas mais adequadas para lidar com o fenômeno. Em outras palavras, deve-se levar em conta que há diferenças entre os desempregados, e as medidas de combate devem ser focadas em grupos específicos.

Além desta introdução, o trabalho está dividido em outras quatro seções. $\mathrm{Na}$ segunda, é apresentada uma breve revisão da literatura sobre a inserção de jovens no mercado de trabalho; na terceira, a metodologia; na quarta, são apresentados os resultados; e na quinta, são apresentadas as principais conclusões.

\footnotetext{
Essa hipótese é semelhante à de Camargo e Reis (2005). No entanto, estes autores utilizaram uma metodologia distinta à deste estudo e não fizeram comparações com o efeito de outras variáveis, como as características individuais e familiares.
} 


\section{Revisão de Literatura}

O desemprego pode ser definido como uma situação na qual o indivíduo deseja estar empregado a uma dada taxa de salário prevalecente no mercado de trabalho, mas não encontra emprego. Já a inatividade é atribuída aos que não trabalham e nem buscaram, ativamente, um trabalho. Não há uma teoria consensual na literatura que explique as causas do desemprego e da inatividade; o que há são hipóteses que tratam de alguns de seus aspectos, ou seja, que procuram responder por que, em determinado instante do tempo, alguns trabalhadores se encontram desempregados ou inativos (ZYLBERSTAJN; BALBINOTTO NETTO, 1999).

Existem, na literatura sobre o mercado de trabalho brasileiro, diversos estudos que procuram entender o desemprego e a inatividade, seja especificamente entre os jovens, seja na população economicamente ativa em geral, abordando questões relativas à sua causa, incidência e duração. Algumas das principais fontes de informações desses estudos são a Pesquisa Mensal de Emprego (PME), a Pesquisa Nacional por Amostra de Domicílios (PNAD) e a Pesquisa de Emprego e Desemprego (PED).

Com respeito à inatividade, Tomás et al. (2008) argumentaram que, no Brasil, tem havido um adiamento da entrada dos jovens no mercado de trabalho. Para esses autores, o prolongamento da inatividade não é explicado apenas pela dedicação aos estudos, uma vez que grande parte dos jovens combina as duas atividades, mas também por dificuldades decorrentes do mercado de trabalho e das mudanças culturais na transição para a vida adulta.

A idade e a experiência têm sido apontadas em diversos estudos, como os de Barros et al. (1997), Fernandes e Picchetti (1999), Menezes-Filho e Picchetti (2000), Silva e Kassouf (2002), Camargo e Reis (2005), entre outros, como as principais causas do desemprego de jovens. De modo geral, pode-se afirmar que esses estudos confirmam que os jovens estão expostos a altas taxas de desemprego, elevada probabilidade de entrada 
no desemprego e alta rotatividade, ou seja, grandes chances de entrada e saída do desemprego.

Quando se trata, especificamente, da experiência, Menezes-Filho e Picchetti (2000) enfatizaram que os que já trabalharam têm probabilidade menor de permanecer desempregados em comparação aos que procuram um emprego pela primeira vez. Camargo e Reis (2005) explicaram esse fato ao argumentarem que a falta de experiência causa um problema de assimetria de informações no mercado de trabalho, dado que parte das características produtivas do trabalhador costuma ser revelada à medida que ele participa do mercado de trabalho. Assim, assimetrias de informações mais elevadas estão associadas a taxas de desemprego maiores, o que é mais problemático para os jovens.

Diversos estudos também sugerem que a relação entre qualificação e taxa de desemprego tem o formato de U-invertido, como os de Amadeo et al. (1994), Barros et al. (1997) e Fernandes e Picchetti (1999). Camargo e Reis (2005) explicaram que a taxa de desemprego entre trabalhadores qualificados é relativamente baixa e sistematicamente decrescente à medida que aumentam os anos de estudo, mas o formato de U-invertido é uma característica dos trabalhadores jovens.

As condições da demanda agregada têm efeito importante ao condicionar a geração de emprego, penalizando todos os trabalhadores, mas principalmente os jovens, que, diante da escassez de oportunidades de emprego, entram em desvantagem na disputa por um posto de trabalho em um mercado cada vez mais exigente. Nesse sentido, Reis e Camargo (2007) destacaram que quedas no nível da atividade econômica também podem explicar o maior desemprego dos jovens em relação aos adultos. Isso ocorre, em geral, quando cai o nível de atividade, e os trabalhadores com menos capital humano específico são demitidos mais facilmente do que aqueles com mais experiência.

Por fim, também é comum na literatura que se recorra a características como cor/raça, gênero e condição socioeconômica dos indivíduos - essa última representada pela variável renda - para explicar sua inserção no 
mercado de trabalho. Quase a totalidade dos estudos citados nessa seção faz uso dessas variáveis. No que se refere à cor e gênero, sua inclusão nos trabalhos objetiva verificar se diferentes grupos raciais, bem como homens e mulheres, apresentam formas distintas de participação no mercado de trabalho. O resultado mais comum é que há discriminação econômica, isto é, mulheres e indivíduos não-brancos têm maiores dificuldades de inserção. Com relação à variável renda, a expectativa teórica é que afete positivamente o salário de reserva dos agentes, contribuindo para o aumento do desemprego.

\section{Metodologia}

\subsection{Especificação econométrica}

Para estudar os fatores determinantes do desemprego e inatividade entre jovens de 16 a 29 anos, será utilizado o modelo econométrico logit multimomial, que é baseado na análise de probabilidades. De acordo com sua especificação ${ }^{7}$, a probabilidade de que um indivíduo i se encontre em determinada situação j (empregado, desempregado ou inativo), condicional a um conjunto de regressores $x_{i}$ (representados por características individuais como gênero, raça, escolaridade, experiência, renda familiar etc.) é dada por

$p_{i j}=\operatorname{Pr}\left(y_{i}=j \mid x_{i}\right)=F_{j}\left(x_{i}, \beta\right), j=1, \ldots, m$ e $i=1, \ldots, N$

em que $y_{i}$ é a variável aleatória que indica a situação em que o indivíduo se encontra com relação ao trabalho, sendo $y_{i}=0$, se for inativo; $y_{i}=1$, se estiver empregado; e $y_{i}=2$, se estiver desempregado; $\beta$ representa o vetor de parâmetros a serem estimados que refletem o impacto das alterações em $x_{i}$ na probabilidade de o indivíduo se encontrar numa das três situações; e $F_{j}($.) é a forma funcional do modelo.

\footnotetext{
O modelo desenvolvido nessa seção foi baseado em Cameron e Trivedi (2005).
} 
Apenas $(m-1)$ probabilidades podem ser estimadas, uma vez que $\sum_{j=1}^{m} p_{i j}=1$. Dessa forma, pode-se representar $F_{m}\left(x_{i}, \beta\right)=1-\sum_{j=1}^{m-1} F_{j}\left(x_{i}, \beta\right)$. Utilizando a função logística e normalizando-a em relação a uma das possíveis situações em que o indivíduo se encontre, tem-se

$$
p_{i j}=\frac{\exp \left(x_{i}^{\prime} \beta_{j}\right)}{1+\sum_{j=1}^{m} \exp \left(x_{i}^{\prime} \beta_{k}\right)}
$$

Em geral, o modelo logit multinomial é estimado pelo método de Máxima Verossimilhança (MV), que pressupõe que as observações da amostra sejam resultado de processos aleatórios independentes e identicamente distribuídos (iid). Tal hipótese, entretanto, não é adequada quando se utilizam dados amostrais complexos, como os da PNAD, utilizados neste estudo; a estimação por MV levaria a estimativas incorretas dos errospadrão e, consequentemente, dos testes de hipótese. Dessa forma, a estimação será feita pelo método da Máxima Pseudo-Verossimilhança (MPV), que possibilitará a obtenção apropriada dos erros padrão ${ }^{8}$.

Em geral, os parâmetros do modelo logit multinomial não são diretamente interpretáveis, portanto, é comum, na literatura, calcular os efeitos marginais $(E M)$. Para um indivíduo $i$, o $E M$ de uma mudança no $k$-ésimo regressor sobre a probabilidade da alternativa $j$ é dado por

$$
E M_{i j k}=\frac{\partial \operatorname{Pr}\left(y_{i}=j\right)}{\partial x_{i k}}=\frac{\partial F_{j}\left(x_{i}, \beta\right)}{\partial x_{i k}}
$$

Ressalta-se que, não necessariamente, o $E M$ terá o mesmo sinal dos coeficientes estimados. Para cada regressor, há $m E M$ s que correspondem

\footnotetext{
Mais detalhes sobre o método de MPV podem ser obtidos em Skinner et al. (1989) e sobre a complexidade dos dados da PNAD, em Silva et al. (2002).
} 
a cada uma das $m$ alternativas e que somam 0 , dado que as probabilidades somam 1 .

\subsection{Base de dados e as variáveis utilizadas}

$\mathrm{Na}$ análise dos fatores determinantes do desemprego e da inatividade de jovens de 15 a 29 anos, foi utilizada a PNAD de 2007. A pesquisa, realizada anualmente (exceto em anos de censo) pelo Departamento de Emprego e Rendimento do Instituto Brasileiro de Geografia e Estatística (IBGE), tem como finalidade produzir informações básicas para o estudo do desenvolvimento socioeconômico do País. A PNAD é baseada em amostra probabilística de domicílios e tem âmbito nacional. Os dados da pesquisa foram coletados em setembro de $2007^{9}$.

Para a criação da variável dependente , que indica a situação em que o indivíduo se encontra em relação ao trabalho, são classificados como ocupados todos os indivíduos de 15 a 29 anos de idade que trabalharam ou tinham emprego na semana de referência da pesquisa - 23 a 29 de setembro de 2007. Como desempregados, foram considerados os indivíduos que não tinham trabalho, mas que tomaram alguma providência para conseguir um emprego na mesma data. Os demais foram classificados como inativos, ou seja, não estavam trabalhando, nem procurando trabalho. Foram considerados apenas moradores das regiões metropolitanas de Belém, Fortaleza, Recife, Salvador, Belo Horizonte, Rio de Janeiro, São Paulo, Curitiba e Porto Alegre. Esse procedimento gerou uma amostra de 34.348 indivíduos. A Tabela 1 apresenta a distribuição dos jovens conforme a situação ocupacional. Embora haja concentração de jovens na categoria empregado, o número de inativos somado ao de desempregados corresponde a cerca de $40 \%$ da amostra.

9 Para mais detalhes sobre a base de dados, consultar IBGE (2008). 
Tabela 1 - Distribuição de frequência relativa dos jovens por situação ocupacional

\begin{tabular}{lcc}
\hline Situação ocupacional & Número de jovens & Freqüência relativa \\
\hline Inativo & 8.930 & 0,26 \\
Empregado & 20.609 & 0,60 \\
Desempregado & 4.809 & 0,14 \\
Total & 34.348 & 100 \\
\hline
\end{tabular}

Fonte: Elaborado pelos autores a partir de dados da PNAD 2007.

As características individuais que podem afetar as probabilidades de desemprego e inatividade, consideradas neste estudo, foram idade, cor ou raça, gênero, escolaridade, experiência, renda familiar e condição do indivíduo na família. Essas variáveis são comumente utilizadas na literatura, como nos estudos de Fernandes e Picchetti (1999), MenezesFilho e Picchetti (2000) e Silva e Kassouf (2002). A seguir, serão descritas cada uma das variáveis que compõem .

- Homem: dummy que terá valor 1 se o indivíduo for do sexo masculino;

- Raça: dummy que terá valor 1 se o individuo for branco;

- Idade: idade do indivíduo;

- Idade2: quadrado da idade;

- Estudante: dummy que terá valor 1 se o indivíduo for estudante e 0 , caso contrário;

- Escolaridade: anos de estudo do individuo;

- Escolaridade2: quadrado dos anos de estudo; 
- Experiência: idade do indivíduo menos a idade com que começou a trabalhar ${ }^{10}$;

- Filho: dummy que terá valor 1 se a posição ocupada na família pelo indivíduo for a de filho;

- Cônjuge: dummy que terá valor 1 se a posição ocupada na família pelo individuo for a de cônjuge;

- Outros: dummy que terá valor 1 se a posição ocupada na família pelo indivíduo não for a de filho, nem cônjuge e nem chefe; $e$

- Renda: renda domiciliar per capita.

\section{Resultados}

Nessa seção, são apresentados e discutidos os principais resultados obtidos no estudo.

Inicialmente, na Tabela 2 são apresentadas as estatísticas descritivas das variáveis explicativas, calculadas para a amostra utilizada nesta pesquisa. Destaca-se que foram apresentadas as médias incondicionais, ou seja, valores baseados em toda a amostra.

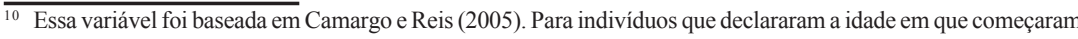
a trabalhar como inferior a 10 anos, a experiência potencial foi calculada pela idade do trabalhador menos 10 . 
Tabela 2 - Estatísticas descritivas das variáveis explicativas

\begin{tabular}{lcccc}
\hline \multicolumn{1}{c}{ Variáveis } & Média & Desvio-Padrão & Mínimo & Máximo \\
\hline Homem & 0,49 & 0,50 & 0 & 1 \\
Raça & 0,46 & 0,50 & 0 & 1 \\
Idade & 22,58 & 3,95 & 16 & 29 \\
Estudante & 0,33 & 0,47 & 0 & 1 \\
Escolaridade & 10,50 & 3,19 & 1 & 17 \\
Experiência & 4,73 & 5,01 & 0 & 19 \\
Filho & 0,53 & 0,50 & 0 & 1 \\
Cônjuge & 0,17 & 0,37 & 0 & 1 \\
Outros & 0,09 & 0,29 & 0 & 1 \\
Renda & 545,81 & 889,10 & 0 & 39.200 \\
\hline
\end{tabular}

Nota: Diferentemente das variáveis contínuas, no caso das dummies são apresentadas as proporções de ocorrência de cada categoria.

Fonte: Resultados da pesquisa.

Observa-se, na Tabela 2, que, dada a amostra formada por indivíduos com idade entre 16 e 29 anos, a média de idade era de, aproximadamente, 23 anos. A proporção de indivíduos do sexo masculino é de $49 \%$ e a de jovens da raça branca é de $46 \%$. Com relação à educação, verificou-se que $33 \%$ dos jovens amostrados eram estudantes e que a média de escolaridade era relativamente alta, de cerca de 11 anos de estudo. Mais de $50 \%$ dos jovens ocupavam a posição de filhos no domicílio, enquanto a posição de cônjuge era de $17 \%$. A renda domiciliar média foi de aproximadamente um salário mínimo, $\mathrm{R} \$ 545,81$. Por fim, no que diz respeito à experiência dos jovens no mercado de trabalho, observou-se uma média de 4,7 anos.

A Tabela 3 contém os coeficientes estimados para o modelo logit multinomial, que foi normalizado em relação à probabilidade de o jovem estar empregado. Ressalta-se que a magnitude dos coeficientes não tem, em geral, significado econômico; todavia, a análise de seus sinais é de 
grande importância para verificar de que maneira as variáveis incluídas no modelo afetam a probabilidade de inatividade e desemprego ${ }^{11}$.

Tabela 3 - Estimativa do modelo logit multinomial e efeitos marginais

\begin{tabular}{|c|c|c|c|c|c|c|c|c|}
\hline & \multicolumn{4}{|c|}{ Inatividade } & \multicolumn{4}{|c|}{ Desemprego } \\
\hline & Coef. & P-valor & EM & P-valor & Coef. & P-valor & EM & P-valor \\
\hline Intercepto & 13,7050 & 0,000 & - & - & 4,2676 & 0,000 & - & - \\
\hline Homem & $-0,4986$ & 0,000 & $-0,0610$ & 0,000 & $-0,2718$ & 0,000 & $-0,0209$ & 0,000 \\
\hline Raça & 0,0005 & 0,989 & 0,0023 & 0,661 & $-0,1018$ & 0,022 & $-0,0119$ & 0,016 \\
\hline Idade & $-1,1804$ & 0,000 & 0,0122 & 0,000 & $-0,5158$ & 0,000 & 0,0192 & 0,000 \\
\hline Idade2 & 0,0291 & 0,000 & - & - & 0,0146 & 0,000 & - & - \\
\hline Estudante & 0,6530 & 0,000 & 0,0912 & 0,000 & 0,1363 & 0,011 & 0,0004 & 0,951 \\
\hline Escolaridade & $-0,1331$ & 0,000 & $-0,0208$ & 0,000 & 0,0960 & 0,004 & $-0,0041$ & 0,000 \\
\hline Escolaridade2 & $-0,0015$ & 0,291 & - & - & $-0,0077$ & 0,000 & - & - \\
\hline Experiência & $-0,4837$ & 0,000 & $-0,0576$ & 0,000 & $-0,3408$ & 0,000 & $-0,0294$ & 0,000 \\
\hline Filho & $-0,0065$ & 0,923 & $-0,0060$ & 0,482 & 0,2398 & 0,001 & 0,0279 & 0,000 \\
\hline Cônjuge & 0,9836 & 0,000 & 0,1455 & 0,000 & 0,4816 & 0,000 & 0,0298 & 0,002 \\
\hline Outros & $-0,0244$ & 0,765 & $-0,0079$ & 0,430 & 0,2050 & 0,026 & 0,0261 & 0,025 \\
\hline Renda & 0,0000 & 0,224 & 0,0000 & 0,000 & $-0,0008$ & 0,000 & $-0,0001$ & 0,000 \\
\hline
\end{tabular}

Fonte: Resultados da pesquisa.

Pode-se observar, inicialmente, de acordo com o p-valor, que todos os coeficientes estimados na equação de desemprego foram estatisticamente significantes a $1 \%$ ou $5 \%$, mas, na de inatividade, cinco variáveis não foram significativas (raça, escolaridade2, filho, outro e renda). Entre as variáveis significativas nos dois casos, seis apresentaram o mesmo sinal, afetando, igualmente, as duas probabilidades.

Os resultados para as variáveis que se referem às características de sexo, raça e idade dos indivíduos estão em conformidade com aspectos amplamente discutidos na literatura. Os jovens do sexo masculino e de cor branca tinham menor probabilidade tanto de estarem inativos quanto

\footnotetext{
${ }_{11}$ Os coeficientes foram estimados pelo método de Máxima Pseudo-Verossimilhança, dado que o plano amostral adotado na PNAD é complexo. Dessa forma, no Anexo são apresentados dois indicadores que avaliam o impacto da incorporação do plano amostral sobre a precisão das estimativas.
} 
desempregados, o que pode ser um indício, segundo Silva e Kassouf (2002), de discriminação no mercado de trabalho brasileiro.

Com relação à idade, as probabilidades de desemprego e de inatividade eram mais elevadas entre os indivíduos mais jovens e passaram a decrescer à medida que envelheciam. E de acordo com Camargo e Reis (2005), principalmente até os 24 anos, as taxas de desemprego são substancialmente altas, até mesmo para indivíduos com elevada qualificação educacional. Além disso, quando se trata especificamente da inatividade, pode-se argumentar que esse resultado confirma a menor intensidade da busca por emprego dos jovens.

Os sinais das variáveis estudante, escolaridade e escolaridade2 mostraram que a probabilidade de desemprego era maior para os que ainda estudavam e crescia com os anos de estudo até atingir um valor máximo (para indivíduos de aproximadamente 6 anos de estudo), quando então passava a decrescer. No caso da inatividade, a probabilidade era maior para os estudantes e decrescia, continuamente, com a educação.

Com relação ao desemprego, esse resultado foi semelhante ao de vários estudos como aqueles desenvolvidos por Amadeo et al. (1994), Barros et al. (1997), Fernandes (199) e Pichetti (1999), e confirmou o padrão de U-invertido entre educação e taxa de desemprego, que, conforme Camargo e Reis (2005), no Brasil é uma característica principalmente dos trabalhadores jovens. Segundo Fernandes e Picchetti (1999), para os indivíduos com pouca instrução, uma parte do crescimento da probabilidade de desemprego com a escolaridade é explicada pelo fato de a participação na força de trabalho também crescer com os anos de estudo.

No que se refere à inatividade, a maior probabilidade para estudantes em função do sinal positivo do coeficiente da variável estudante - pode ser um indício a favor do argumento deste estudo, isto é, que os jovens têm dedicado cada vez mais seu tempo à qualificação e adiado sua entrada no mercado de trabalho. No entanto, o decréscimo da probabilidade com 
os anos de estudo indica que, à medida que vão atingindo certo nível educacional, a tendência é optar pela inserção na PEA.

A variável experiência tem importância fundamental neste estudo, principalmente com relação à hipótese assumida para explicar o desemprego. O sinal negativo confirma que quanto maior a experiência, menor a probabilidade de desemprego (e também de inatividade). Para Camargo e Reis (2005), à medida que o trabalhador for acumulando experiência, mais informações serão reveladas sobre as suas características produtivas e, dessa forma, mais chances terá de conseguir um emprego.

Entre as variáveis que representam a posição ocupada pelo jovem na família (filho, cônjuge, outros e chefe, sendo essa última a que foi utilizada como base), apenas a variável cônjuge foi estatisticamente significativa na equação de inatividade. O coeficiente positivo indica que os cônjuges tinham maior probabilidade de estar fora do mercado de trabalho em comparação com os chefes de família. Com relação à probabilidade de desemprego, as três variáveis foram estatisticamente significativas e tiveram efeitos marginais muito semelhantes; o sinal positivo de seus coeficientes sugere que os chefes de família tinham menor probabilidade de estar desempregados. Como, em geral, os chefes são os responsáveis pela totalidade ou maior parte do orçamento doméstico, sua busca por emprego tenderia a ser mais intensa e persistente.

A variável referente à renda familiar foi estatisticamente significativa apenas para a probabilidade de desemprego. O sinal negativo do coeficiente não era esperado, dado que rendas maiores tenderiam a aumentar o salário de reserva dos indivíduos e, portanto, também elevar o salário exigido para aceitar um emprego, fazendo com que, ceteris paribus, fosse maior a probabilidade de desemprego.

Todavia, esse resultado, aparentemente controverso, não é incomum na literatura, e diferentes justificativas são apresentadas pelos autores. Fernandes e Picchetti (1999) argumentaram que a queda na probabilidade de desemprego poderia estar relacionada ao aumento da inatividade com 
o suporte financeiro; Silva e Kassouf (2002) e Fernandes et al. (2008) acreditavam que, quanto maior a renda familiar, maiores as condições de o jovem ter formação educacional e preparo para o mercado de trabalho, aumentando, assim, sua empregabilidade, tudo o mais constante.

Os efeitos marginais de cada variável, calculados em seus respectivos pontos médios, permitiram fazer algumas considerações a respeito das hipóteses testadas neste estudo. Verificou-se, pela sua magnitude (em módulo), que as variáveis mais expressivas para explicar a inatividade foram estudante e cônjuge e, para o desemprego, experiência e cônjuge. Entre os demais EMs, alguns não foram estatisticamente significativos ou apresentaram valores muito baixos e semelhantes.

Com relação à inatividade, pode-se afirmar que o adiamento da entrada do jovem no mercado de trabalho não era condicionado, exclusivamente, pelo aumento da escolaridade. Embora o fato de estudar aumente, em média, nove pontos percentuais, a probabilidade de inatividade do jovem, é a condição de cônjuge a principal determinante, segundo os resultados deste estudo (aumenta em 14 pontos percentuais). Esse resultado sugere, como argumentado por Tomás et al. (2008), que a inatividade pode encobrir outro lado das atividades juvenis, como, por exemplo, a realização de tarefas domésticas, que, em geral, são executadas pelo cônjuge e por outros membros da família, que não o chefe. Esses autores ressaltaram ainda que a inatividade não poderia ser justificada apenas pela dedicação aos estudos, já que, no Brasil, os jovens não necessariamente entram para o mercado de trabalho somente após terminarem os estudos.

No caso do desemprego, os resultados indicam uma possível não rejeição da hipótese do estudo. De acordo com o valor do efeito marginal, cada ano adicional de experiência reduziria a probabilidade de o jovem estar desempregado em, aproximadamente, três pontos percentuais. Pode-se afirmar que quanto mais experiência tiver o trabalhador, maior será a quantidade de informações que os empregadores terão a respeito de sua produtividade e, dessa forma, menor será sua dificuldade de inserção no mercado de trabalho. No entanto, não se pode fazer uma inferência categórica, uma vez que a variável Cônjuge apresentou o valor do efeito 
marginal muito próximo ao da variável Experiência, indicando que também teve importância expressiva para explicar o desemprego de jovens no período estudado.

Por fim, ao comparar o valor do efeito marginal da escolaridade e da experiência, observa-se que o segundo é superior, tanto para a inatividade quanto para o desemprego. Segundo Silva e Kassouf (2002), esse resultado indica que o mercado de trabalho tem valorizado mais a experiência do jovem. Tendo em vista que na faixa etária estudada, em geral, os jovens têm pouca experiência, os que têm certa vantagem nesse aspecto tendem a estar em melhor situação que os demais.

\section{Conclusões}

Embora seja expressiva a presença de jovens no mercado de trabalho brasileiro, a inatividade e o desemprego são fenômenos que ainda atingem, principalmente, essa parcela da população, quando comparados a outros grupos etários. A exclusão dos jovens do mercado de trabalho pode comprometer seu desempenho socioeconômico futuro e contribuir para agravar os problemas sociais do país. Nesse sentido, este estudo procurou analisar os principais determinantes do desemprego e da inatividade de jovens de 16 a 29 anos de idade nas principais regiões metropolitanas brasileiras.

De modo geral, pode-se concluir que jovens do sexo feminino, não brancos, com menor grau de escolaridade e experiência e que ocupam a posição de cônjuge na unidade familiar, têm maior probabilidade de estar na situação de inativo ou desempregado. A variável renda familiar, que, em muitos estudos, é apontada como um dos principais determinantes da inserção no mercado de trabalho, não apresentou resultados expressivos neste trabalho.

Deve-se destacar que, quando o jovem ocupa a posição de cônjuge na família, sua probabilidade de pertencer à categoria dos inativos é bastante 
aumentada em relação à posição de chefe. Esse resultado tende a ser especialmente condizente com a realidade quando se consideram as dificuldades que as mulheres jovens e que são casadas enfrentam para participar da força de trabalho, dado que acabam por se dedicar, quase que exclusivamente, à realização de tarefas domésticas.

Outro resultado do estudo que merece destaque é o fato de que quanto mais jovem for o trabalhador e menor sua experiência, maior será sua dificuldade de inserção no mercado de trabalho. Isso ocorre porque as informações que os empregadores têm sobre a produtividade dos trabalhadores mais jovens são limitadas quando comparados àqueles que acumularam mais experiência no mercado de trabalho.

Esses resultados sugerem a necessidade de formular políticas públicas que visem a preparar melhor os jovens para sua inserção no mercado de trabalho. É preciso adotar medidas que garantam acesso à educação de qualidade, já que a probabilidade de desemprego tende a diminuir à medida que aumenta a educação, assim como criar programas para facilitar a obtenção do primeiro emprego e, assim, adquirir experiência, já que esta é tão valorizada pelos empregadores.

Por fim, deve-se ressaltar uma limitação do estudo, ao considerar, em conjunto, jovens do sexo masculino e feminino, pois é grande a discussão de que há diferenças significativas entre a forma com a qual homens e mulheres se inserem no mercado de trabalho. Dessa forma, sugere-se, para trabalhos posteriores, que análises comparativas sejam realizadas. 


\section{Referências}

AMADEO, E.; CAMARGO, J. M.; GONZAGA, G.; BARROS, R. P.; MENDONÇA, R. S. P. A natureza e o funcionamento do mercado de trabalho brasileiro desde 1980. Rio de Janeiro: IPEA, 1994 (Texto para Discussão, 353).

BARROS, R.; CAMARGO, J. M.; MENDONÇA, R. Estrutura do desemprego no Brasil. Rio de Janeiro: IPEA, 1997 (Texto para Discussão, 478).

BRAGA, T. S.; RODARTE, M. M. S. A inserção ocupacional e o desemprego dos jovens: o caso das Regiões Metropolitanas de Salvador e Belo Horizonte. Ensaios FEE, Porto Alegre, v. 26, n. 2, p. 877-904, 2005.

CACCIAMALI, M. C; BRAGA, T. S. A armadilha social destinada aos jovens: mercado de trabalho insuficiente, oferta educacional restrita e de baixa qualidade e ações públicas incipientes. In: CACCIAMALI, M. C CHAHAD, J. P. Z. (orgs.). Mercado de trabalho no Brasil: novas práticas trabalhistas, negociações coletivas e direitos fundamentais do trabalho. São Paulo. LTR, 2003. p. 469-500.

CAMARGO, J. M.; REIS, M. C. Desemprego: o custo da desinformação. Revista Brasileira de Economia, v. 59, n. 3, p. 381-5425, 2005.

CAMERON, L. C.; TRIVEDI, P. K. Microeconometrics: methods and applications. New York, USA: Cambridge University Press, 2005. $1034 \mathrm{p}$.

DEPARTAMENTO INTERSINDICAL DE ESTATÍSTICA E ESTUDOS SOCIOECONÔMICOS - DIEESE. A ocupação dos jovens nos mercados de trabalho metropolitanos. Estudos e Pesquisas, n. 24, 2006. Disponível em: http://www.mp.rs.gov.br/areas/infancia/arquivos/ dieese.pdf. Acesso em: 25/05/2009. 
FERNANDES, R. A. S.; LIMA, J. E.; SANTOS, C. M. A exclusão social de mulheres jovens, com idade entre 15 a 24 anos, no mercado de trabalho no Brasil. Revista de Economia e Administração, v. 7, n. 2, p. 125-136, 2008.

FERNANDES, R.; PICCHETTI P. Uma análise do desemprego e da inatividade no Brasil metropolitano. Pesquisa e Planejamento Econômico, v. 29, n.1. p. 87-112, 1999.

INSTITUTO BRASILEIRO DE GEOGRAFIA E ESTATÍSTICA IBGE. Pesquisa Nacional por Amostra de Domicílios 2007: notas metodológicas - pesquisa básica. Rio de Janeiro: IBGE. 2008. 57p.

INSTITUTO DE PESQUISA ECONÔMICA APLICADA - IPEA. PNAD 2007 - Primeiras Análises: educação, juventude, raça/cor. Comunicado da Presidência, n. 12, 2008. Disponível em: http:// www.ipea.gov.br/sites/000/2/comunicado_presidencia/ Comunicado_\%20da_\%20presidencian12.pdf. Acesso em 25/05/2009.

INSTITUTO DE PESQUISA ECONÔMICA APLICADA - IPEA. PNAD 2009 - Primeiras Análises: o mercado de trabalho brasileiro em 2009. Comunicados do IPEA, n. 62, 2010. Disponível em: http:// www.ipea.gov.br/portal/images/stories/PDFs/comunicado/ 100923_comunicadoipea62.pdf. Acesso em 15/11/2011.

KORENMAN, S.; NEUMARK, D. Cohort crowding and youth labor markets: a cross-national analysis. NBER Working Paper, 6031. 1997.

LAYARD, R.; NICKELL, S.; JACKMAN, R. Unemployment: Macroeconomic Performance and the Labour Market. Oxford: Oxford University Press. 1991

MENEZES-FILHO, N.; PICCHETTI, P. Os determinantes da duração do desemprego em São Paulo. Pesquisa e Planejamento Econômico, v. 30, n. 1, p. 23-48, 2000. 
POCHMANN, M. Situação do jovem no mercado de trabalho no Brasil: um balanço dos últimos 10 anos. Disponível em: <http:// w w w . cursodevera ofortaleza.com.br/2007/ Textos / Situa $\%$ E7\%E3o\%20do\%20Jovem\%20no\%20mercado\%20de\%20trabalho.pdf.> Acesso em: 03/08/2009.

REIS, M. C.; CAMARGO, J. M. Desemprego dos jovens no Brasil: os efeitos da estabilização da inflação em um mercado de trabalho com escassez de informação. Revista Brasileira de Economia, v. 61, n. 4, p. 493-518, 2007.

SILVA, N. D. V.; KASSOUF A. L. A exclusão social dos jovens no mercado de trabalho brasileiro. Revista Brasileira de Estudos de População, v. 19, n. 2, p. 99-115, 2002.

SILVA, P. L. N.; PESSOA, D. G. C.; LILA, M. F. Análise de dados da PNAD: incorporando a estrutura do plano amostral. Ciência \& Saúde Coletiva, v. 7, n. 4, p. 659-670, 2002.

SKINNER, C. J. HOLT, D.; SMITH, T. M. F. Analysis of complex surveys. Chichester, UK: John Wiley \& Sons Inc. 1989. 328 p.

ZYLBERSTAJN, H.; BALBINOTTO NETTO, G. As teorias de desemprego e as políticas públicas de emprego. Estudos Econômicos, v. 29, n. 1, p. 129-149, 1999.

TOMÁS, M. C.; OliveirA, A. M. H. C.; RIOS-NETO, E. L. G. Adiamento do ingresso no mercado de trabalho sob o enfoque demográfico: uma análise das regiões metropolitanas brasileiras. Revista Brasileira de Estudos de População, v. 25, n. 1, p. 91-107, 2008. 


\section{Anexo}

Na Tabela A1 são apresentados os coeficientes estimados do modelo logit multinomial, os mesmos da Tabela 2, juntamente com o cálculo de dois indicadores que avaliam o impacto da incorporação do plano amostral sobre a precisão das estimativas: Efeito do Plano Amostral (DEFF Design Effect) e Efeito do Plano Amostral Ampliado (MEFF Misspecification Effect $)^{12}$. Essas medidas fornecem um indicativo da importância de se considerarem as características do plano amostral, uma vez que permitem observar em quanto a estimativa da variância dos coeficientes seria subestimada ou superestimada caso o plano amostral complexo e suas peculiaridades sejam ignorados.

As estimativas do efeito do plano amostral indicaram que sua correta especificação foi fundamental para a obtenção de estimativas robustas e não viciadas. Todas as estimativas DEFF e MEFF foram superiores a 1 , indicando que as variâncias das estimativas dos coeficientes seriam subestimadas caso a amostra complexa fosse considerada como uma amostra aleatória simples.

12 Detalhes sobre os cálculo desses indicadores podem ser obtidos em Skinner et al. (1989). 
Dênis Antônio da Cunha, Aracy Alves de Araújo \& João Eustáquio de Lima

Tabela A1 - Estimativas dos coeficientes para as equações de emprego e desemprego e do efeito do plano amostral

\begin{tabular}{|c|c|c|c|c|c|c|}
\hline & \multicolumn{3}{|c|}{ Inatividade } & \multicolumn{3}{|c|}{ Desemprego } \\
\hline & Coef. & DEFF & MEFF & Coef. & DEFF & MEFF \\
\hline Intercepto & 13,7050 & 1,5199 & 1,5145 & 4,2676 & 1,3602 & 1,3478 \\
\hline Homem & $-0,4986$ & 1,5277 & 1,3846 & $-0,2718$ & 1,4903 & 1,4685 \\
\hline Raça & 0,0005 & 1,4920 & 1,4319 & $-0,1018$ & 1,4638 & 1,4279 \\
\hline Idade & $-1,1804$ & 1,5104 & 1,5349 & $-0,5158$ & 1,3575 & 1,3501 \\
\hline Idade 2 & 0,0291 & 1,4978 & 1,5485 & 0,0146 & 1,3595 & 1,3732 \\
\hline Estudante & 0,6530 & 1,5390 & 1,3614 & 0,1363 & 1,5233 & 1,5079 \\
\hline Escolaridade & $-0,1331$ & 1,3183 & 1,4122 & 0,0960 & 1,2980 & 1,5574 \\
\hline Escolaridade2 & $-0,0015$ & 1,3239 & 1,4317 & $-0,0077$ & 1,3037 & 1,5207 \\
\hline Experiência & $-0,4837$ & 1,4985 & 2,7154 & $-0,3408$ & 1,3734 & 2,0550 \\
\hline Filho & $-0,0065$ & 1,3126 & 1,4074 & 0,2398 & 1,3984 & 1,4996 \\
\hline Cônjuge & 0,9836 & 1,3641 & 1,5933 & 0,4816 & 1,3954 & 1,5607 \\
\hline Outros & $-0,0244$ & 1,2102 & 1,2468 & 0,2050 & 1,3602 & 1,4730 \\
\hline Renda & 0,0000 & 1,2846 & 1,5733 & $-0,0008$ & 1,6442 & 3,0234 \\
\hline
\end{tabular}

Fonte: Resultados da pesquisa. 
REVISTA DE ECONOMIA E AGRONEGÓCIO, VOL.9, $N^{\circ} 3$ 